\title{
IMPORTANCE OF HISTOPATHOLOGY IN A CASE OF TUBEROUS SCLEROSIS COMPLEX
}

\author{
Jehaan Wadia ${ }^{1}$, Haritosh Velankar ${ }^{2}$, Yogesh Dabhokar ${ }^{3}$
}

1Postgraduate Resident, Department of ENT, Dr. D. Y. Patil Hospital and Research Centre, Nerul, Navi Mumbai.

2 Professor, Department of ENT, Dr. D. Y. Patil Hospital and Research Centre, Nerul, Navi Mumbai.

3 Professor and HOD, Department of ENT, Dr. D. Y. Patil Hospital and Research Centre, Nerul, Navi Mumbai.

HOW TO CITE THIS ARTICLE: Wadia J, Velankar H, Dabhokar Y. Importance of histopathology in a case of tuberous sclerosis complex. J. Evolution Med. Dent. Sci. 2017;6(67):4829-4830, DOI: 10.14260/Jemds/2017/1048

\section{PRESENTATION OF CASE}

A 26-year-old male presented with diffuse nodular growth over the face and nose in butterfly pattern. The tumours were causing nasal blockage, profuse bleeding, diminished vision and inability to eat solid foods. The facial tumours were insidious in onset and progressed to the current stage causing significant disability. Patient had been diagnosed during childhood with intellectual disability. The patient also had a history of developmental delay and behavioural abnormalities. There was history of recurrent seizures since childhood, which had been aggravated recently. Patient also complained of haematuria since 2 months.

The patient was admitted under ICU care for airway management and fluid imbalance. Serum electrolytes were deranged and serum creatinine was noted to be raised markedly suggestive of renal failure, for which patient was started on dialysis.

Patient had difficulty in swallowing solids and was on a liquid diet. RT insertion was attempted to support oral intake, but attempts of insertion resulted in excessive bleeding from the ruptured nodules in and around the nose. Patient was put on TPN to support oral intake.

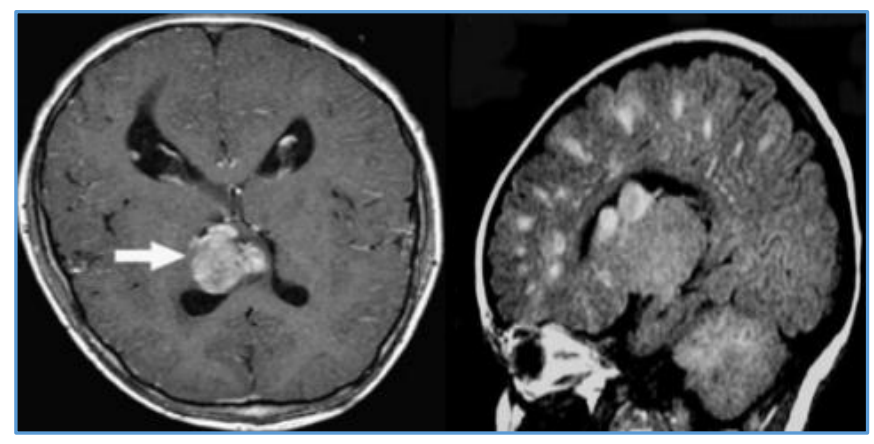

Figure. 1 Brain Imaging shows Subependymal Giant Cell

Financial or Other, Competing Interest: None.

Submission 29-06-2017, Peer Review 23-07-2017,

Acceptance 29-07-2017, Published 21-08-2017.

Corresponding Author:

Dr. Jehaan Wadia,

22, Bakhtawar,

Narayan Dabholkar Road,

Mumbai- 400006,

Maharashtra.

E-mail: jehaanwadia@gmail.com DOI: $10.14260 /$ jemds $/ 2017 / 1048$

(c) $(1) \Theta$

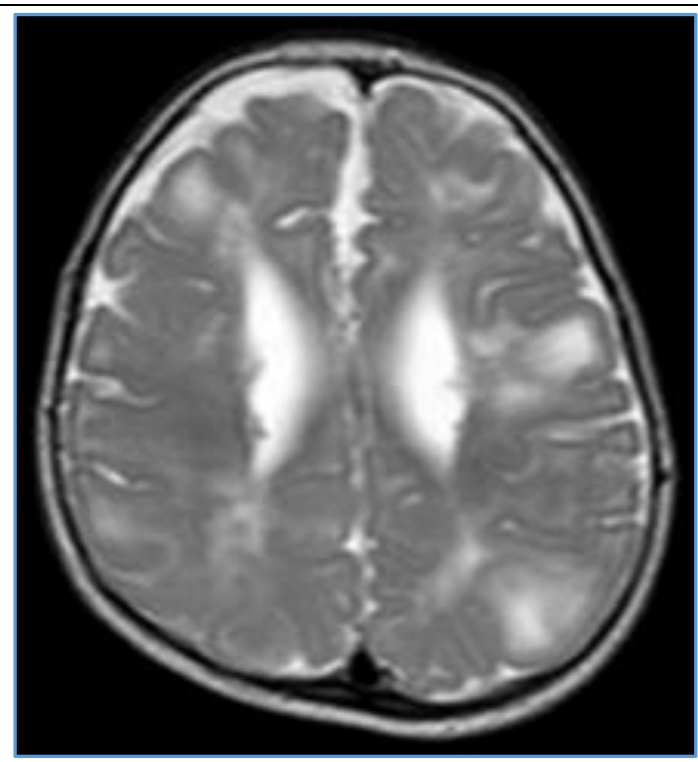

Figure 2. MRI Brain showing Subcortical Tubers and Subependymal Nodules

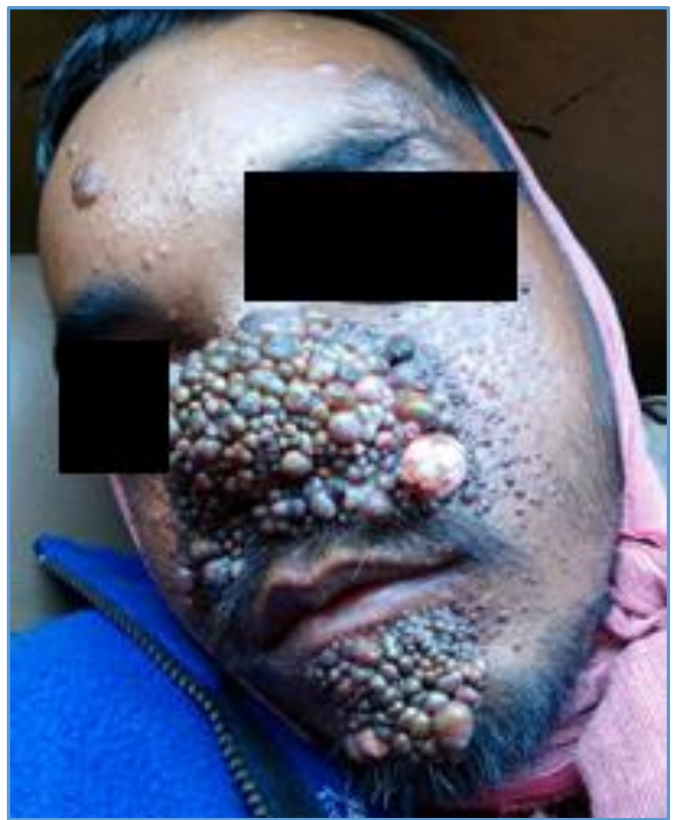

Figure 3. Nodular Lesions over the Face and Nose

\section{CLINICAL DIAGNOSIS}

Recurrent episodes of profuse bleeding from nodules over nose and face had resulted in major blood loss. The patient received blood transfusion and IV fluids, but the haemoglobin level only raised from $6.4 \mathrm{mg} / \mathrm{dL}$ to $7.2 \mathrm{mg} / \mathrm{dL}$. Active bleeds from the nodules were controlled either by direct pressure, cautery or nasal packing. A biopsy was taken from the lesions over the face and vestibule of the nose and sent for histopathology, which revealed nodular sclerosis. 
Imaging of brain showed presence of cortical tubers and nodular, band-like lesions seen in white matter. Imaging of the kidneys showed bilateral, multiple and large angiomyolipomas.

As per the clinical and radiological findings along with the histopathology report, a comprehensive diagnosis of tuberous sclerosis complex was made.

Clinical diagnosis of TSC is made by the physician based on clinical features of mental disability, classical facial lesions etc. and imaging findings, which may show tumours in the brain, kidney etc. As there is a wide variation in presentation and symptoms of these cases, a swift diagnosis is not always easy. Correlation of clinical features with histopathology and imaging findings along with a high index of suspicion is essential to make this difficult diagnosis.

\section{DISCUSSION OF MANAGEMENT}

Tuberous sclerosis is a neurocutaneous disorder characterised by the development of multiple benign tumours of the embryonic ectoderm. It is an autosomal dominant disease occurring equally in all races and ethnic groups and equally in both sexes. The disease usually affects 7 - 12 out of every 100000 people.(1) Many of these cases went undetected until the advent of Ultrasound Sonography, Computed Tomography and Magnetic Resonance Imaging.

This case report is presented to underscore the importance of testing the lesions and correlating the test results with clinical features and detailed history. The comprehensive diagnosis could have been missed had a biopsy of the nodule and consequent histopathology testing not been done.

\section{CONCLUSION}

TSC habitually affects multiple organ systems and can exhibit differently in individual patients and also in different stages of life. Medications, surgery and other interventions can be effective in managing some of the symptoms of TSC.(2)

Antiepileptics may be prescribed for control of seizures and other medications for behavioural disabilities depending on severity.

Everolimus, the immunosuppressant drug has been used in cases of TSC involving subependymal giant cell tumours and renal angiomyolipomas.

Surgery may be attempted in some cases to control seizures or limit blood loss from nodules over face.
Embolisation is another technique used to treat bleeding renal tumours.

Prognosis for persons with TSC depends on the severity of manifestations, which can range from mild skin lesions to varying grades of learning disabilities and epilepsy to severe intellectual disability, uncontrollable seizures, profuse bleeding from facial nodules and even kidney or lung failure. Those individuals with mild symptoms can generally live long productive lives, whereas individuals with the more severe form may have serious disabilities.(3)

Leading causes of death include renal disease, brain tumour, lymphangioleiomyomatosis of the lung, and status epilepticus or bronchopneumonia in those with severe mental handicap. ${ }^{(4)}$

Approximately $40 \%$ of patients die by age 35 from complications of one or more of the manifestations.(5) Diagnosis of the disease should be followed by genetic counselling.

Awareness regarding different organ manifestations of TSC is important, so as to not miss out on the correct diagnosis and approach.

\section{ACKNOWLEDGEMENTS}

We would like to acknowledge the Doctors and Nursing staff in our department and hospital, who participated in the treatment of this patient.

Thanks to Dr. Rajendra Joshi (Consulting ENT surgeon, Bilaspur) for referring the patient.

\section{REFERENCES}

[1] O'Callaghan FJ, Shiell AW, Osborne JP, et al. Prevalence of tuberous sclerosis estimated by capture-recapture analysis. Lancet 1998;351(9114):1490.

[2] Samir H, Ghaffar HA, Nasr M. Seizures and intellectual outcome: clinico-radiological study of 30 Egyptian cases of tuberous sclerosis complex. Eur J Paediatr Neurol 2011;15(2):131-7.

[3] Tuberous sclerosis fact sheet. National institute of neurological disorders and stroke 2006.

[4] Shepherd CW, Gomez MR, Lie JT, et al. Causes of death in patients with tuberous sclerosis. Mayo Clin Proc 1991;66(8):792-6.

[5] Umeoka S, Koyama T, Miki Y, et al. Pictorial review of tuberous sclerosis in various organs. RadioGraphics 2008;28(7):e32. 